\title{
Percepção de Fala e Limiares Audiométricos em Usuários de Implante Coclear Nucleus 22 e Nucleus 24
}

\section{Speech Perception and Audiometry Thresholds in Nucleus Cochlear 22 and Nucleus 24 Implant Users}

\author{
Paola Angelica Samuel*, Maria Valéria Schmidt Goffi Gomez**, Débora Maria Befi Lopes ***, Carla Gentile \\ Matas ****, Robinson Koji Tsuji*****, Rubens Vuono de Brito Neto******, Ricardo Ferreira Bento*******. \\ * Fellow em Implante Coclear do Hospital das Clínicas da Faculdade de Medicina da Universidade de São Paulo, HC-FMUSP, São Paulo, SP. Fonoaudióloga da Equipe de \\ Implante Coclear do Hospital das Clínicas da Faculdade de Medicina da Universidade de São Paulo, HC-FMUSP, São Paulo, SP. \\ ** Doutora em Distúrbios da Comunicação Humana (Fonoaudiologia) pela Universidade Federal de São Paulo. Fonoaudióloga; Hospital das Clínicas da Faculdade de Medicina \\ da Universidade de São Paulo, HC-FMUSP, São Paulo, SP. \\ *** Livre Docência pela Universidade de São Paulo. Fonoaudióloga; Professora Associada da Universidade de São Paulo. \\ **** Doutoura em Distúrbios da Comunicação Humana pela Universidade Federal de São Paulo. Fonoaudióloga; Professora adjunta da Universidade de São Paulo.

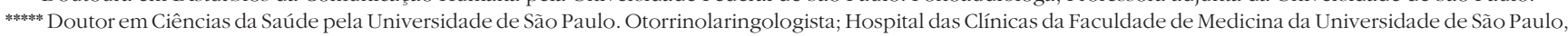 \\ HC-FMUSP, São Paulo, SP. \\ ******: Livre Docência pelo Hospital das Clínicas da Faculdade de Medicina da Universidade de São Paulo, HC-FMUSP. Otorrinolaringologista; Hospital das Clínicas da Faculdade \\ de Medicina da Universidade de São Paulo, HC-FMUSP, São Paulo, SP. \\ ********* Livre Docência pela Universidade de São Paulo. Otorrinolaringologista; Hospital das Clínicas da Faculdade de Medicina da Universidade de São Paulo, HC-FMUSP, \\ São Paulo, SP.
}

Instituição: Programa de Aprimoramento em Implante Coclear do Departamento de Fisioterapia, Fonoaudiologia e Terapia Ocupacional da Universidade de São Paulo, e Equipe de Implante Coclear do Hospital das Clínicas da Faculdade de Medicina da Universidade de São Paulo. São Paulo / SP - Brasil.

Endereço para correspondência: Paola Angelica Samuel - Rua Capote Valente, 432 - Conjunto 14 - Pinheiros - São Paulo / SP - Brasil - CEP: 05409-001 - Telefone: (+55 11) 3898-2210 - E-mail: paolasamuel@yahoo.com.br

Artigo recebido em 22 de Abril de 2010. Artigo aprovado em 10 de Junho de 2010.

\section{RESUMO}

Introdução:

Objetivo:

Método:

Resultados:

Conclusão:

Palavras-chave:

\section{SUMMARY}

Introduction:

Objective:

Method:

Results:

Conclusion:

Keywords:
Clinicamente observa-se contribuição da tecnologia do implante coclear (IC) Nucleus 24 (N24). A confirmação dessa evidência seria importante pois afeta diretamente os critérios de indicação do implante coclear.

Verificar a contribuição da atualização da tecnologia do IC sobre limiares audiométricos e desempenho na percepção de sentenças após 12 meses de uso do implante.

Estudo retrospectivo de corte transversal, com coleta em banco de dados. Critérios de seleção: idade igual ou superior a 18 anos, uso do dispositivo de IC N22 ou N24, tempo de privação auditiva até 20 anos, cuja etiologia da surdez não seja meningite. A amostra foi dividida em G22 (usuários do N22), e G24 (usuários do N24). Os grupos foram comparados em relação aos resultados no teste de percepção de sentenças no silêncio e limiares audiométricos após 12 meses de uso.

Dos 84 pacientes adultos selecionados, 54 preencheram os critérios de seleção, sendo 13 usuários de N22 e 41 usuários de N24. Não houve diferença estatisticamente significante entre resíduo auditivo pré-operatório e tempo de privação auditiva dos usuários do N22 e N24. Os usuários do N24 apresentaram melhores médias nos limiares audiométricos, mas os testes de percepção de sentenças no silêncio não indicaram diferença entre modelos.

A contribuição da tecnologia foi evidenciada apenas nos limiares audiométricos. Novos estudos estão sendo conduzidos para avaliar a contribuição da tecnologia na percepção de fala em situações auditivas mais difíceis. implante coclear, audição, perda auditiva, testes auditivos, percepção da fala.

Clinically it was observed the contribution of the Nucleus 24 (N24) cochlear implant (CI) technology. The confirmation of this evidence would be important because it affects directly the indication criteria of the cochlear implant.

To verify the contribution of the technology's updating of the CI about the auditory thresholds and the performance in the perception of sentences after 12 mouths of implant use.

A retrospective, cross-sectional study with database collection. Selection criteria: age equal or higher than 18 years old, CI N22 or N24 device use, auditory privation time until 20 years, which deafness etiology does not be meningitis. The sample was divided into G22 (N22 users), and G24 (N24 users). The groups were compared concerning the result in the sentences perception test in the silence and audiometric thresholds after 12 mouths of use.

From 84 adults patients selected, 54 filled the selection criteria, being 13 users of N22 and 41 users of N24. There was no difference statistically significant between preoperative auditory residues and time of auditory privation of the N22 and N24 users. The N24 users presented best averages in the audiometric thresholds, but the sentences perception tests in the silence do not indicated difference between models.

The contribution of the technology was evidenced only in the auditory thresholds. News studies are being performed in order to evaluate the technology contribution in the speech perception in auditory situations more difficult. 


\section{INTRODUÇÃO}

A surdez profunda é uma deficiência que afeta diretamente o indivíduo e as pessoas ao seu redor, pois prejudica sua comunicação, personalidade e relacionamento com os outros (1).

O uso de aparelhos de amplificação sonora individual (AASI) pode beneficiar indivíduos portadores de deficiência auditiva de diversos graus (de leve a profundo). Porém, por ser um amplificador sonoro, necessita de uma reserva coclear suficiente para que possa haver uma boa percepção do som e, principalmente, da fala. Alguns indivíduos, entretanto, apresentam uma disfunção auditiva muito importante, e não conseguem obter benefícios com o uso do AASI. Esses indivíduos, então, podem ser candidatos ao Implante Coclear (1,2). Os implantes cocleares tradicionalmente são indicados para os pacientes cuja perda auditiva não permite um ganho funcional suficiente para percepção de fala com as próteses auditivas convencionais (3).

O implante coclear (IC) é um sistema capaz de restaurar funcionalmente o sistema sensorial auditivo. (1,4). Desde o lançamento do IC multicanal Nucleus 22 por Graeme Clark em 1978 (5) existe a preocupação de incrementar a tecnologia. Dentre as diferentes marcas de implantes cocleares multicanais, estão os modelos Nucleus 22 (N22) e Nucleus 24 (N24).

O N22 é um implante coclear multicanal formado por um componente interno (composto por receptor/ estimulador, e feixe com 22 eletrodos), pelo processador de fala modelo Spectra $22^{\circledR}$, e pela antena transmissora. O processador de fala tem a função de converter a informação transmitida pelo microfone em estímulos elétricos que representem aspectos da fala que possam ser percebidos pelo paciente. Cada modelo de implante coclear possui uma estratégia de processamento da fala para alcançar esse objetivo $(6,7)$.

No N22, a estratégia de processamento da fala utilizada é a Spectral Peak (SPEAK). Esta seleciona uma média de 6-8 frequências a partir de um filtro de 20 bandas de frequências. Estas frequências selecionadas, denominadas máximas, são apresentadas aos eletrodos de uma forma não simultânea, a uma velocidade de estimulação média de $250 \mathrm{~Hz}$ para minimizar interações entre os eletrodos (6).

Com os avanços tecnológicos, o modelo Nucleus $22^{\circledR}$ foi gradativamente substituído pelo modelo Nucleus $24^{\circledR}$, também desenvolvido pela Cochlear ${ }^{\circledR}$. O Nucleus 24 (N24) é um implante coclear multicanal, formado por um componente interno (composto por receptor/ estimulador, feixe com 24 eletrodos, sendo dois eletrodos extra-cocleares, utilizados em diferentes modos de estimulação), por dois diferentes modelos de processador de fala (modelo SPrint, no formato de 'caixinha' utilizada pelo usuário, e modelo ESPrit, no formato de processador retroauricular), e antena transmissora.

O N24 possui a opção de diferentes estratégias de processamento de fala: SPEAK, Continuous Interleaved Sample (CIS) e Advanced Encoder Conversion (ACE).

A estratégia ACE combina as vantagens das estratégias SPEAK e CIS. O espectro de frequência é dividido em 22 canais para estimulação. O número de máximas selecionado é constante e depende do valor especificado na programação do processador de fala. Da mesma forma que a CIS, a estratégia ACE possui maior velocidade de estimulação se comparada à SPEAK, variando entre $500 \mathrm{e}$ $2400 \mathrm{~Hz}$ (8).

Waltzman et al., 1999 (9) realizaram um estudo comparando o desempenho de usuários de implante coclear N22 e N24, em testes de percepção de fala apresentados em formato aberto. Os autores utilizaram 16 usuários de N22, e 20 usuários de N24, todos com a estratégia de codificação de fala SPEAK. A média de idade dos usuários de N22 era maior que a do N24, assim como o tempo de privação auditiva. Concluíram que a percepção de fala de usuários de N22 e N24 é semelhante até os três meses de uso; após os 3 meses, usuários de N22 não apresentaram evolução significante em seu desempenho, enquanto os usuários de N24 continuaram evoluindo. Os autores ressaltam a importância de realizar mais estudos, principalmente comparando diferentes formas de estimulação e estratégias de codificação de fala.

BRITo, 2000 (2) estudou os resultados auditivos e a qualidade de vida de 10 pacientes usuários do implante coclear modelo N22. Após seis meses de uso, com a estratégia de processamento de fala SPEAK, os pacientes apresentaram média dos limiares audiométricos em 42,7 dBNA, 82,8\% de acertos no teste de reconhecimento de sentenças em apresentação em formato fechado, e 56\% de acertos em apresentação de sentenças em formato aberto. O questionário de qualidade de vida após o implante mostrou que houve percepção de melhora na qualidade de vida pelos familiares dos usuários do IC.

BENTO et al., 2004 (1) analisaram os resultados auditivos em 61 usuários de implante coclear, com tempo mínimo de uso de seis meses. A média dos limiares audiométricos foi de 38,7 dBNPS, enquanto a média em reconhecimento de sentenças em formato aberto foi de $71,3 \%$, vogais em $86,5 \%$, monossílabos em $52,60 \%$ e consoante medial em 52,6\%. A maioria dos pacientes estava apta 
a usar o telefone. Os autores concluíram que os pacientes obtiveram um excelente resultado em testes de reconhecimento de palavras e sentenças em apresentação aberta, readquirindo uma audição útil. Entretanto, não houve a preocupação de separação das tecnologias nesse trabalho.

Temos como objetivo neste estudo verificar se há contribuição da tecnologia do N24 sobre limiares audiométricos e desempenho na percepção de sentenças, quando comparados com o N22, após 12 meses de uso.

\section{MÉTOdo}

\section{Procedimentos}

Trata-se de um estudo retrospectivo de corte transversal, com coleta em banco de dados, realizado no Programa de Aprimoramento em Implante Coclear do Ambulatório de Implante Coclear do Hospital das Clínicas da Faculdade de Medicina da Universidade de São Paulo (HCFMUSP), tendo sido autorizado pelo responsável, e aprovado pelo Comitê de Ética da Instituição sob o protocolo 633/04.

Os critérios de seleção da amostra foram:

- Idade igual ou superior a 18 anos, idade em que uma pessoa é considerada adulta (10).

- Uso do dispositivo de Implante Coclear modelos Nucleus 22 ou Nucleus 24 (Cochlear Ltd - Austrália.)

- Uso sistemático do IC (uso por pelo menos oito horas diárias, durante 12 meses consecutivos)

- Dados equivalentes aos testes de percepção de sentenças e limiares audiométricos no período de 12 meses de uso do IC.

Dos pacientes selecionados de acordo com os critérios acima, foram excluídos os pacientes:

- Cuja etiologia da surdez seja meningite.

- Com tempo de privação auditiva maior ou igual a 20 anos.

Foram analisados dados dos prontuários de todos os pacientes submetidos à cirurgia de Implante Coclear na Instituição, operados no período de janeiro de 1999 a maio de 2007. Os prontuários foram selecionados de acordo com a idade, e analisados em dois grupos, de acordo com o modelo do IC: Grupo 22 (G22) - usuários do N22, e Grupo 24 (G24) - usuários do N24. Os grupos foram analisados em subgrupos de acordo com o tempo de privação auditiva: de zero a 10 anos, e de 11 a 20 anos.

Foram coletados dados da avaliação pré-operatória e com o IC após 12 meses de uso dos limiares audiométricos em campo livre nas frequências de $500 \mathrm{~Hz}, 1 \mathrm{kHz}, 2 \mathrm{kHz}$, $4 \mathrm{kHz}, 6 \mathrm{kHz}$ e $8 \mathrm{kHz}$, e resultados dos testes de percepção de sentenças em apresentação aberta e fechada em ambiente silencioso (11). Os limiares ausentes foram considerados como 130dB.

O teste de percepção de sentenças em apresentação aberta é realizado apenas auditivamente, sem apoio de gestos ou leitura labial. O teste realizado em apresentação fechada também é realizado apenas auditivamente, porém o paciente possui o apoio visual em forma escrita de múltipla escolha das sentenças que serão utilizadas. Estes testes são baseados no Protocolo Latino-Americano (12) para avaliação do IC, e fazem parte do Protocolo HCFMUSP (3).

Os grupos foram comparados em relação aos resultados no teste de percepção de sentenças e média dos limiares audiométricos. Para a comparação destas variáveis entre os grupos G22 e G24, foi utilizado o teste não paramétrico de Mann-Whitney. Para a comparação entre as idades dos pacientes e o tempo de privação, foi utilizado o teste t-Student para amostras independentes. Foram consideradas diferenças estatisticamente significantes quando os valores de $\mathrm{p}<0,05$.

\section{RESULTADOS}

Dos 84 pacientes adultos com etiologia não-meningite implantados com os modelos N22 e N24, 54 pacientes preencheram os critérios de seleção, sendo 13 usuários de N22 e 41 usuários de N24.

O tempo de privação auditiva entre os grupos foi estatisticamente semelhante, assim como as idades dos usuários do G22 e G24 (Tabela 1).

Não houve diferença estatisticamente significante entre o resíduo auditivo pré-operatório dos usuários do IC N22 e N24 (Figura 1).

Nos limiares audiométricos após um ano de uso do IC, houve diferença estatisticamente significante entre os grupos G22 e G24, sendo os limiares de G24 melhores do que a mesma variável em G22 (Figura 2).

Tabela I. Comparação da idade e tempo de privação auditiva entre os usuários nos grupos G22 e G24.

\begin{tabular}{lccc}
\hline & \multicolumn{2}{c}{ Grupos } & \\
& $G 22(n=13)$ & $G 24(n=41)$ & -valor \\
\hline Idade & $38,9 \pm 9,8$ & $39,2 \pm 9,8$ & 0,93 \\
Tempo de privação & & & \\
auditiva & $6,7 \pm 5,9$ & $7,0 \pm 6$ & 0,90 \\
\hline
\end{tabular}


Nos testes de percepção de sentenças no silêncio, não houve diferença estatisticamente significante entre G22 e G24 em apresentação aberta e fechada (Figura 3).

\section{DISCUSSÃO}

Nossa amostra totalizou 54 sujeitos, sendo 13 usuários do N22 e 41 usuários do N24. Esta diferença na amostra deve-se a que o N22 foi usado nos anos de 1999, 2000 e 2001, sendo substituído pelo N24 a partir de 2001.

Excluímos pacientes cuja etiologia da perda auditiva tenha sido meningite, pois esta é considerada preditora para um baixo desempenho com o IC, devido à redução do número de células do gânglio espiral, característica da lesão no sistema auditivo causada pela meningite $(4,13)$.

Foram selecionados os pacientes cujo tempo de privação auditiva fosse entre zero e 20 anos, evitando assim interferência dessa variável nos resultados. Alguns autores referem que um longo tempo de privação auditiva pode influenciar negativamente nos testes de percepção de fala $(9,13,14,15)$. Porém, o tempo de privação auditiva entre os dois grupos foi muito homogêneo, e por isso não interferiram nas variáveis estudadas.

Clinicamente, observamos a contribuição da tecnologia do N24 sobre o N22. No entanto, é necessário confirmar esta contribuição, visto que afeta diretamente os critérios de indicação do implante coclear.

Nossos resultados mostraram diferença estatisticamente significante na audiometria dos usuários de N22 e N24, sendo que as médias das frequências de 500 a $4000 \mathrm{~Hz}$, de $6000 \mathrm{~Hz}$ e $8000 \mathrm{~Hz}$ foram menores nos usuários do N24, indicando melhor desempenho na audiometria. Estes resultados corroboram a literatura (16), que também encontraram melhores limiares audiométricos em usuários de N24 quando comparados ao N22. A contribuição dos limiares auditivos pode ter implicação na percepção de sons de fraca energia acústica e em intensidades baixas $(17,18)$.

Ao mesmo tempo, foi importante a homogeneidade das amostras no que se refere ao resíduo auditivo préoperatório. Como os critérios audiológicos estão evoluindo com a tecnologia $(13,19)$, existiria a dúvida de que os pacientes do G24 pudessem ser favorecidos por maior resíduo pré-operatório.

SkinNer et al. (1999b) (17) avaliaram a percepção de fala de oito adultos pós-linguais, usuários do IC N22 com a estratégia SPEAK, de acordo com os níveis mínimos de estimulação. Os níveis mínimos foram fixados num valor

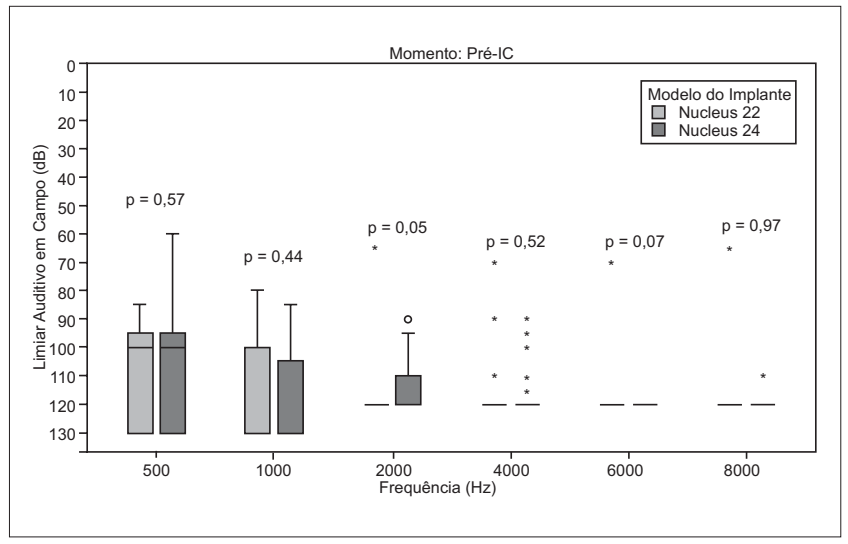

Figura 1. Comparação entre os limiares audiométricos préimplante dos usuários dos grupos G22 e G24.

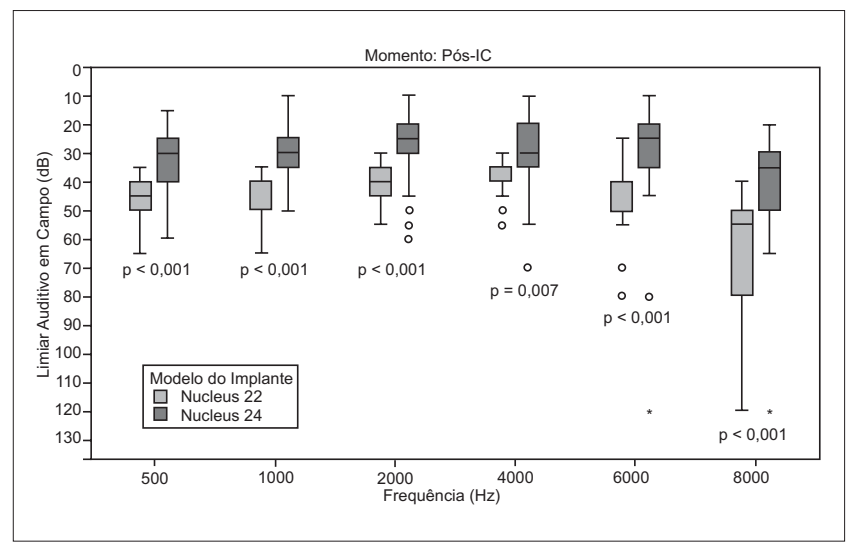

Figura 2. Comparação dos limiares audiométricos pós-implante entre os usuários dos grupos G22 e G24.

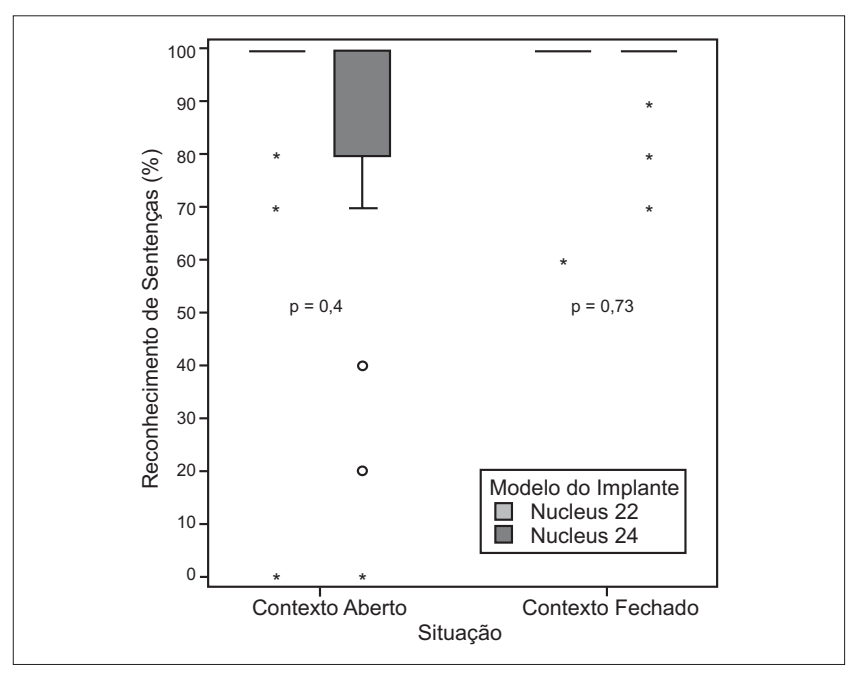

Figura 3. Comparação do desempenho em testes de percepção de sentenças em ambiente silencioso entre os usuários dos grupos G22 e G24. 
limite (valor padrão clínico) e níveis mais elevados (aproximadamente $+2.04 \mathrm{~dB}$ ), para determinar se o aumento dos níveis melhoraria a compreensão dos sons. Os usuários do IC N22 participaram da pesquisa realizada em quatro fases. A percepção de fala foi avaliada com palavras de núcleo consoante-vogal-consoante no silêncio, e com sentenças no ruído, ambos apresentados a 50, 60 e 70 dB NPS durante duas sessões semanais no fim de cada fase. A pontuação média dos participantes foi maior na fase de maior quantidade de palavras e fonemas, apresentados a 50 e $60 \mathrm{~dB}$ NPS, e nas sentenças apresentadas de 50 a $70 \mathrm{~dB}$ NPS. Todos os participantes optaram por usar o programa com níveis mais elevados no cotidiano, ao final do estudo. Os resultados sugerem que o uso clínico de um programa com níveis mais elevados no N22 facilita a compreensão dos sons no dia a dia. Mais estudos são necessários para determinar se a abordagem é adequada para outros modelos do implante coclear.

Na percepção de sentenças, não foram observadas diferenças estatisticamente significantes entre usuários de N22 e N24, porém a média de desempenho dos usuários do N22 foi maior que dos usuários do N24. WaLTZman et al., 1999 (8) concluíram que a percepção de fala em usuários do N22 e N24 é similar, porém acreditavam que após 12 meses de uso, a tecnologia poderia beneficiá-los na percepção de fala.

Na nossa amostra, a mediana do G22 nos testes de percepção de sentenças já era de 100\%, ou seja, o bom desempenho dos pacientes influenciou o resultado estatístico. A aplicação de testes mais difíceis, como palavras compostas por 'vogal-consoante-vogal', poderia mudar o desempenho dos pacientes.

Os usuários de N22 na nossa amostra utilizaram a estratégia de processamento de fala SPEAK, enquanto todos os usuários do N24 utilizaram a estratégia de processamento de fala ACE.

PSARros et al., 2002 (18) compararam o desempenho de sete crianças usuárias do N24, durante a transição do uso da estratégia SPEAK, utilizada por pelo menos seis meses, para a estratégia ACE. No estudo, a maior diferença estabelecida entre as estratégias foi a velocidade de estimulação ( $250 \mathrm{~Hz}$ para SPEAK, e $900 \mathrm{~Hz}$ para ACE). Após a mudança, não houve piora na percepção de fala, mas algumas crianças tiveram uma dificuldade maior no início do uso da estratégia ACE. Os autores concluíram que houve uma melhora na percepção de fala das crianças, e que os pacientes podem se beneficiar ao alternar as duas estratégias de codificação de fala em diferentes situações de escuta.

SKINNER et al., 2002 (20) compararam o desempenho de 62 indivíduos usuários das estratégias SPEAK, ACE e CIS, todos usuários de N24, por meio da aplicação de listas de palavras, sentenças, teste de consoante medial e vogais. Os usuários experimentaram as diferentes estratégias, e os autores concluíram que a estratégia ACE apresentou melhor desempenho em testes de percepção de fala.

Romero et al., 2004 (21), estudando resultados auditivos em indivíduos pós-linguais usuários de implante coclear, referiram que entre seis e 12 meses de uso do implante coclear, alguns pacientes obtém desempenho máximo nesse período, enquanto outros continuam evoluindo após os 12 meses de uso.

Em relação à influência do tempo de privação auditiva na percepção de fala, não foram encontradas diferenças estatisticamente significantes entre os grupos divididos de acordo com o tempo de privação. Nos usuários do N22, o desempenho no teste de percepção de sentenças em conjunto fechado foi semelhante, independente do tempo de privação auditiva, discordando dos achados descritos na literatura $(13,15)$. Estes autores relataram que o tempo de privação auditiva é diretamente proporcional ao menor desempenho na percepção de fala.

No nosso estudo, todos os testes de percepção de fala utilizados foram aplicados em situação de silêncio. Situações ruidosas são mais desafiadoras para o usuário de implante coclear, visto que reduzem significantemente o índice de reconhecimento de fala. No entanto, o treinamento das habilidades auditivas e o conhecimento de estratégias que minimizem os efeitos negativos do ruído na comunicação são de extrema importância (22). Grande parte dos estudos realizados com percepção de fala foi realizada em situações de silêncio (23). Este estudo nos levou a escolher protocolos de avaliação que incluam testes em situações mais desafiadoras e que refletiriam o dia a dia dos pacientes.

BRito Neto, 2000 (2) referiu que enquanto a avaliação auditiva constitui um critério objetivo de avaliação do paciente antes e depois do implante coclear, ela não refletiu sozinha o resultado da condição de ouvinte para os pacientes, antes surdos. As condições sócio-econômicas e culturais dos pacientes implantados e, portanto, suas diferentes necessidades de uso da audição tornam necessária uma avaliação que leve em conta as situações e ambientes acústicos, características de seu estilo de vida e de seu relacionamento com a família e comunidade.

\section{CONCLUSÃO}

Os usuários do IC N24 apresentaram melhores médias nos limiares audiométricos, quando comparados aos usuários do N22, mas os testes de percepção de fala 
realizados não indicaram diferença entre os modelos. Não houve diferença entre os testes de percepção de sentenças e limiares audiométricos quando relacionados ao tempo de privação auditiva, exceto no limiar de $8000 \mathrm{~Hz}$ em usuários de N22. Quando a intenção for determinar a contribuição da tecnologia, estudos devem ser realizados, com amostras maiores e com testes realizados em situações mais desafiadoras (por exemplo, fala no ruído), para verificar se a tecnologia influencia também os resultados na percepção de fala.

\section{REFERÊNCIAS BIBLIOGRÁFICAS}

1. Bento RF, Brito RV, Castilho AM, Goffi Gomez MVS, Giorgi SB, Guedes MC. Resultados auditivos com o implante coclear multicanal em pacientes submetidos a cirurgia no Hospital das Clínicas da Faculdade de Medicina da Universidade de São Paulo. Rev Bras Otorrinolaringol. 2004, 70(5):632-7.

2. Brito RV. Estudo dos resultados auditivos e da qualidade de vida em pacientes com implante coclear multicanal. São Paulo, 2000. (Tese de Doutorado - Faculdade de Medicina da Universidade de São Paulo).

3. Goffi-Gomez MVS, Guedes MC, Sant'Anna SBG, Peralta CGO, Tsuji RK, Castilho AM, Brito RV, Bento RF. Critérios de seleção e avaliação médica e audiológica dos candidatos ao implante coclear: Protocolo HCFMUSP. Arq Otorrinolaringol. 2004, 7(3):197-204.

4. Guedes MC, Brito RV, Goffi-Gomez MVS, Sant'Anna SBG, Peralta CGO, Castilho AM, Bento RF. Telemetria de resposta neural intra-operatória em usuários de implante coclear. Rev Bras Otorrinolaringol. 2005, 71(5):660-7.

5. Clark GM, Tong YC, Bailey QR, Black RC, Martin LF, Milar JB, O'Loughlin BJ, Patrick JF, Pyman BC. A multiple-electrode cochlear implant. J Oto-Laryngol Soc. Aust. 1978, 4:208212.

6. Skinner MW, Fourakis MS, Holden TA, Holden LK, Demorest ME. Identification of speech by cochlear implant recipients with the multipeak (MPEAK) and spectral peak (SPEAK) speech coding strategies II. Consonants. Ear and Hear. 1999, 20(6):443-60.

7. Skinner MW, Holden LK, Holden TA, Demorest ME. Effect of stimulation rate on cochlear implant recipients' thresholds and maximum acceptable loudness levels. J Am Acad Audiol. 2000, 11(4):203-13.

8. Skinner MW, Holden LK, Whitford LA, Plant KL, Psarros C, Holden TA. Speech recognition with the Nucleus 24
SPEAK, ACE and CIS speech coding strategies in newly implanted adults. Ear Hear. 2002, 23:207-223.

9. Waltzman SB, Cohen NL, Roland JT. A comparison of the growth of open-set speech perception between the Nucleus 22 and Nucleus 24 cochlear implant systems. The American J Otol. 1999, 20:435-41.

10. Brasil, Casa Civil, 2003. Estatuto do Idoso. Disponível em: http://www.planalto.gov.br/ccivil/LEIS/2003/ L10.741.htm.

11. Costa MJ, Iorio MCM, Mangabeira-Albernaz PL. Desenvolvimento de um teste para avaliar a habilidade de reconhecer a fala no silêncio e no ruído. Pró-fono. 2000, 12(2):09-16.

12. Protocolo Latino Americano para Avaliação de candidatos ao Implante Coclear. Reunião do Grupo de Investigação Latino-Americano da Cochlear Corporation. Bogotá, Colômbia, 2000.

13. Green KMJ, Bhatt YM, Mawman DJ, O'Driscoll MP, Saeed SR, Ramsden RT, Green MW. Predictors of audiological outcome following cochlear implantation in adults. Cochlear Implants Int. 2007, 8(1):1-11.

14. Lyxell B, Andersson J, Arlinger S, Bredberg G, Harder H, RönnbergJ. Verbal information-processing capabilities and cochlear implants: implications for preoperative predictors of speech understanding. J Deaf Stud Deaf Education 1:3 Summer, 1996.

15. Fallon JB, Irvine DRF, Sheperd RK. Cochlear implants and brain plasticity. Hear Res. 2008, 238:110-17.

16. Goffi-Gomez MVS, Guedes MC, Peralta CGO, Tsuji RK, Neto RVB, Bento RF. Limiares auditivos em campo livre em usuários de implante coclear Nucleus 22 e Nucleus 24. Anais do VI Congresso da Fundação Otorrinolaringologia - FORL, 2007.

17. Skinner MW, Holden LK, Holden TA, Demorest ME. Comparison of two methodsfor selecting minimumstimulation levels used in programming the Nucleus 22 cochlear implant. J Speech Lang Hear Res. 1999, 42(4):814-28.

18. Psarros CE, Plant KL, Lee K, Decker JA, Whitford LA, Cowan RSC. Conversion from the SPEAK to the ACE strategy in children using the Nucleus 24 cochlear implant system: speech perception and speech production outcomes. Ear Hear. 2002, 23:18S-27S.

19. David EE, Ostroff JM, Shipp D, Nedzelski JM, Chen JM, Parnes LS, Zimmerman K, Schramm D, Seguin C. Speech 
coding strategies and revised cochlear implant candidacy: an analysis of post-implant performance. Otol Neurotol. 2003, 24(2):228-33.

20. Skinner MW, Arndt PL, Staller SJ. Nucleus 24 Advanced Encoder Conversion study: performance versus preference. Ear Hear. 2002, 23:2S-17S.

21. Romero MJP, Quevedo MS, Segura CR. Cochlear implant in postlingual adults with progressive hearing loss. Acta Otorrinolaringol Esp. 2004, 55:457-462.
22. Nascimento LT, Bevilacqua MC. Avaliação da percepção da fala com ruído competitivo em adultos com implante coclear. Rev Bras Otorrinolaringol. 2005, 71(4):432-8.

23. Bishop R, Littman T, Balko K, Watson S, Backous D. Speech understanding in noise with post-lingual adult cochlear implant users: a comparison of devices. Cochlear Impl Int. 2003, 4(supplement 1):4-5. 